\title{
Lack of Low-mass Meteoroids in the Earth's Meteoroid Flux: \\ Due to Space Erosion?
}

\author{
by
}

David Parry Rubincam

Geodesy and Geophysics Laboratory, Code 61A

Earth Sciences Division

NASA Goddard Space Flight Center

Building 34, Room S280

Greenbelt, MD 20771

voice: $301-614-6464$

fax: 301-614-6522

email: david.p.rubincam@,nasa.gov 


\begin{abstract}
The Earth's cumulative meteoroid flux, when plotted on a log flux vs. log mass graph, has a shallower slope for meteoroid masses less than $\sim 2.5 \mathrm{~kg}$ compared to those with masses greater than $\sim 2.5 \mathrm{~kg}$, indicating a lack of low-mass objects. The lack may be due to a few centimeters of space erosion in a single population, rather than being due to two different meteoroid populations as proposed by Halliday et al. (1996).
\end{abstract}




\section{Introduction}

Halliday et al. $(1989,1996)$ show the Earth's cumulative meteoroid flux $N(>M)$ as a function of meteoroid mass $M$ on a $\log -\log$ plot, where $N(>M)$ is measured in number of fireballs per Earth year. The plot of Halliday et al. (1996) (AA in their Fig. 1) for asteroidal meteoroids in the range $10^{-1} \mathrm{~kg} \leq M \leq \sim 10 \mathrm{~kg}$ is shown here as the heavy broken line in Fig. 1. The plot is a straight line for masses between $\sim 2.5 \mathrm{~kg}$ and $10 \mathrm{~kg}$, and another straight line but with a shallower slope for masses between $10^{-1} \mathrm{~kg}$ and $~ 2.5$ kg. (Bland and Artemieva (2006, their Fig. 1) show Halliday et al.'s data as the upper part of their curve.) The dashed lines in Fig. 1 extend Halliday et al.'s straight lines beyond their data.

Halliday et al. (1996) believe that the change in slope is real, indicating a lack of meteoroids at low mass. They suggest that the break in slope is due to two different meteoroid populations.

The present paper suggests instead that the change in slope is caused by space erosion within a single population. The erosion of meteoroids from the ceaseless impacts of interplanetary dust is an old topic (Whipple and Fireman, 1959; Fisher, 1961; Whipple, 1962; Schaeffer et al., 1981; Hughes, 1982; Wieler and Graf, 2001, p. 227; Welten et al., 2001; Rubincam, 2015; Wiegert, 2015).

Two simple models of space erosion are investigated here. Both models show a bend in the cumulative flux curve near $2.5 \mathrm{~kg}$, with erosion in the range of $0.025-0.050 \mathrm{~m}$ fitting the data fairly well; in other words, erosion of a few centimeters. 


\section{First space erosion model}

The meteoroid mass distribution $f(M)$ due to mutual collisions is initially taken to be

$f(M) d M=\frac{f_{0}}{M^{2}} d M$

without any erosion having taken place. Here $f_{0}$ is a constant and $M$ is the meteoroid mass. Dohnanyi (1969) derives 1.837 as the exponent on $M$, but this is rounded up to 2 here for ease of analytical integration. Moreover, integrating (1) to get the cumulative flux gives a slope of -1 , which agrees with the slope of Halliday et al.'s (1996) data for the more massive meteoroids (rightmost heavy line in Fig. 1).

The meteoroids are assumed to be stony and spherical in shape so that

$M=4 \pi \rho R^{3} / 3$,

where $\rho=2800 \mathrm{~kg} \mathrm{~m}^{-3}$ and $R$ is the radius. In (2) the meteoroid's radius shrinks from abrasive space erosion (Gault et al., 1972) with time according to

$R=R_{0}-|\dot{R}| t$ 
where $R_{0}$ is the meteoroid's radius at time $t=0$ and $\dot{R}$ is the rate at which the radius changes with time. Rubincam (2015) indicates that $\dot{R}$ is constant for an unchanging dust environment. The constancy of $\dot{R}$ is assumed here. (See also Rubincam submitted, 2016).

The first model assumes that erosion takes place only after the distribution (1) is established. For instance, collisions with little erosion produce (1) in the main asteroid belt and then all erosion taking place on the way to Earth, as resonances pump up the meteoroids' orbital eccentricities (Gladman et al., 1997). The first model further assumes that all meteoroids suffer the exact same erosional change in radius $\Delta R$ before reaching Earth with radius $R$. The cumulative distribution will then be

$$
\begin{aligned}
& N_{1}(>M)=A_{0} \int_{M}^{M_{2}} \frac{1}{M^{2}} d M=A_{0}\left(\frac{3}{4 \pi \rho}\right)^{2}(4 \pi \rho) \int_{R}^{R_{2}} \frac{s^{2}}{(s+\Delta R)^{6}} d s \\
& \approx\left(\frac{A_{0}}{M}\right)\left[\frac{1}{(1+c)^{5}}\right]\left[1+\left(\frac{c}{2}\right)+\left(\frac{c^{2}}{10}\right)\right]
\end{aligned}
$$

after using $d M=4 \pi \rho R s^{2} d s$ and setting $c=\Delta R / R$. The integral is evaluated with the aid of Gradshteyn and Ryzhik (1980, p. 58).

The result for $\Delta R=0.025 \mathrm{~m}=2.5 \mathrm{~cm}$ is shown as squares in Fig. 1, where the amplitude $A_{0}$ has been adjusted so that the squares approximate the straight lines. The squares show a bend in the curve at $M \approx 2.5 \mathrm{~kg}$ as in Halliday et al. (1996) and follow the two solid lines quite well over the range of their data. The squares also follow the $1 / M$ 
law for large masses, because $c \rightarrow 0$ as $R \rightarrow \infty$ in (4). At low masses beyond the range of the data, the slope becomes shallower and shallower.

\section{Second space erosion model}

Like the first model, the second model assumes that space erosion takes place only after (1) is established. Unlike the first model in which each meteoroid eroded by the same amount $\Delta R$, the second model assumes that some percentage of masses in the range $R+\Delta R_{1}$ to $R+\Delta R_{2}$ shrink down into the range $R \pm d R / 2$, where $\Delta R_{1}$ and $\Delta R_{2}$ are constants. This is illustrated in Fig. 2. In the upper grey region, a certain fraction $\kappa$ of meteoroids with radii between $R+\Delta R_{1}$ and $R+\Delta R_{2}$ shrink down to radius $\sim R$, as shown in the lower grey region. The same $\kappa$ is assumed to hold for all meteoroids at all radii. The numerical value of $\kappa$ is unimportant; it is absorbed into other constants as shown below. The number of meteoroids in $d R$ is proportional to

$$
\int_{M_{1}}^{M_{2}} \frac{1}{M^{2}} d M=\left(\frac{3}{4 \pi \rho}\right)^{2}(4 \pi \rho) \int_{R+\Delta R_{1}}^{R+\Delta R_{2}} \frac{s^{2}}{s^{6}} d s=\left(\frac{3}{4 \pi \rho}\right)\left[\frac{1}{\left(R+\Delta R_{1}\right)^{3}}-\frac{1}{\left(R+\Delta R_{2}\right)^{3}}\right] .
$$

The cumulative flux for all meteoroids between radii $R$ and $R_{2}$ is proportional to

$$
\int_{R}^{R_{2}}\left[\frac{1}{\left(R+\Delta R_{1}\right)^{3}}-\frac{1}{\left(R+\Delta R_{2}\right)^{3}}\right] d R=\frac{1}{2}\left[\frac{1}{\left(R+\Delta R_{1}\right)^{2}}-\frac{1}{\left(R_{2}+\Delta R_{2}\right)^{2}}\right] .
$$


Hence

$$
N_{2}(>M) \approx\left(\frac{A_{1}}{R^{2}}\right)\left[\frac{1}{\left(1+c_{1}\right)^{2}}-\frac{1}{\left(1+c_{2}\right)^{2}}\right]=\left(\frac{A_{0} R}{M}\right)\left[\frac{1}{\left(1+c_{1}\right)^{2}}-\frac{1}{\left(1+c_{2}\right)^{2}}\right]
$$

assuming $R_{2} \gg R$. Here $A_{1}$ and $A_{0}$ are constants which $\kappa$ is absorbed into, while $c_{1}=$ $\Delta R_{1} / R$ and $c_{2}=\Delta R_{2} / R$.

The circles in Fig. 1 show the results for $\Delta R_{1}=0.025 \mathrm{~m}$ and $\Delta R_{2}=0.050 \mathrm{~m}$, while the crosses are for $\Delta R_{1}=0.050 \mathrm{~m}$ and $\Delta R_{2}=0.100 \mathrm{~m}$. As with the squares, $A_{0}$ is adjusted so that both the circles and the crosses approximate the data. And as with (4), (7) approaches the $1 / M$ law as the masses become large: as $R \rightarrow \infty$, the right side of (7) tends to

$$
\begin{aligned}
& \frac{A_{0} R}{M}\left[\frac{1}{\left(1+c_{1}\right)^{2}}-\frac{1}{\left(1+c_{2}\right)^{2}}\right] \approx \frac{A_{0} R}{M}\left(1-2 c_{1}-1+2 c_{2}\right) . \\
& =\frac{A_{0} R}{M}\left(\frac{\Delta R_{2}}{R}-\frac{\Delta R_{1}}{R}\right)=A_{0}\left(\frac{\Delta R_{2}-\Delta R_{1}}{M}\right) .
\end{aligned}
$$

Also like the first model, the slope becomes shallower and shallower as $M \rightarrow 0$.

\section{Discussion}

The two simple models suggest space erosion may be in the range of $0.025-0.100$ m. How do the above results tally with the erosion rates found in Rubincam (submitted, 
2016)? For meteoroids in prograde circular orbits, a meteoroid's radius erodes at the rate $\dot{R}_{\text {retro }}$ due to impacts from the retrograde dust population (Wiegert, 2015). The rate of shrinkage is approximately

$$
\left|\dot{R}_{\text {retro }}\right|=0.0074\left(\frac{s}{0.5}\right)\left(\frac{2800 \mathrm{~kg} \mathrm{~m}^{-3}}{\rho}\right)\left(\frac{3 \mathrm{AU}}{a}\right)^{5 / 2} \mathrm{~m} \mathrm{My}^{-1},
$$

where $a$ is the meteoroid's orbital semimajor axis, $s$ is the fraction of dust in retrograde orbits, and $\rho$ is the meteoroid's density. Nominal values are taken to be $s=0.5$ (Wiegert, 2015) and $\rho=2800 \mathrm{~kg} \mathrm{~m}^{-3}$.

Meteoroids will be in elliptical orbits while on their way to Earth, and not in circular orbits as assumed in (9). However, using $a=2 \mathrm{AU}$ in (9) probably gives a reasonable estimate for the overall erosion rate. Using this value for semimajor axis in (9) gives a change in radius

$\Delta R=0.2 \mathrm{~m}$

after a meteoroid lifetime of $10^{7} \mathrm{y}$. This is somewhat greater than that found above, but seems reasonably in line with the results found here.

The two models investigated here are crude, but they are suggestive. Both indicate that abrasive space erosion of a few centimeters within a single population may be the reason for the change in slope near $2.5 \mathrm{~kg}$ in the cumulative meteoroid flux, rather than being due to two distinct meteoroid populations. Furthermore, both models predict a 
further flattening out of the slope at lower and lower masses beyond the scope of Halliday et al.'s (1996) data. How much these features would change with a more sophisticated model remains to be seen.

\section{References}

Bland, P. A., Artemieva, N. A., 2006. The rate of small impacts on Earth. Meteorit. Planet. Sci. 41, 607-631.

Dohnanyi, J. S., 1969. Collisional model of asteroids and their debris. J. Geophys. Res. 74, 2531-2554. doi: 10.1029/JB074i010p02531.

Fisher, D. E., 1961. Space erosion of the Grant meteorite. J. Geophys. Res. 66, 15091511. doi: 10.1029/JZ066i005p01509.

Gault, D. E., Hörz, F., Hartung, J. B., 1972. Effects of microcratering on the lunar surface. Proc. Third Lunar Sci. Conf., Vol. 3, pp. 2713-2734.

Gladman, B. J., Migliorini, F., Morbidelli, A., Zappala, V., Michel, P., Cellino, A., Froeschle, C., Levison, H. F., Bailey, M., and Duncan, M., 1997. Dynamical lifetimes of objects injected into asteroid belt resonances. Science 277, 197-201. doi: 10.1126/science.277.5323.197.

Gradshteyn, I., S., Ryzhik, I. M., 1980. Table of Integrals, Series, and Products. Academic Press, New York.

Halliday, I., Blackwell, A T., Griffin, A. A., 1989. The flux of meteorites on the Earth's surface. Meteoritics 24, 173-178. 
Halliday, I., Griffin, A. A., Blackwell, A. T., 1996. Detailed data for 259 fireballs from the Canadian network and inferences concerning the influx of large meteoroids. Meteorit. Planet. Sci. 31, 185-217. doi: 10.1111/j.1945-5100.1996.tb02014.x.

Hughes, D. W., 1982. Meteorite erosion and cosmic ray variation. Nature 295, 279-280. doi: $10.1038 / 295279 \mathrm{a} 0$.

Rubincam, D. P., 2015. Space erosion and cosmic ray exposure ages of stony meteorites. Icarus 245, 112-121. http://dx.doi.org/10.1016/j.icarus.2014.09.005.

Shaeffer, O. A., Nagel, K., Fechtig, H., Neukum, G., 1981. Space erosion of meteorites and the secular variation of cosmic rays (over $10^{9}$ years). Planet. Space Sci. 29, 11091118. doi: 10.1016/0032-0633(81)90010-6.

Welten, K. C., Nishiizumi, K., Caffee, M. W., Schultz, L., 2001. Update on exposure ages of diogenites: The impact and evidence of space erosion and/or collisional disruption of stony meteoroids. Meteoritics \& Planet. Sci. 36, supplement, A223.

Wiegert, P. A., 2015. Meteoroid impacts on asteroids: A competitor for Yarkovsky and YORP. Icarus 252, 22-31. https://dx.doi.org/10.1016/j.icarus.2014.12.022.

Wieler, R., Graf, T., 2001. Cosmic ray exposure: History of meteorites. In: PeuckerEhrenbrink, B., Schmitz, B. (Ed.s), Accretion of Extraterrestrial Matter Throughout Earth’s History. Kluwer Academic/Plenum Publishers, New York, pp. 221-240.

Whipple, F. L., 1962. Meteorite erosion in space. Astron. J. 67, 285. doi: $10.1086 / 108864$

Whipple, F. L., Fireman, E. L., 1959. Calculation of erosion in space from the cosmic-ray exposure ages of meteorites. Nature 183, 1315. doi:10.1038/1831315a0. 


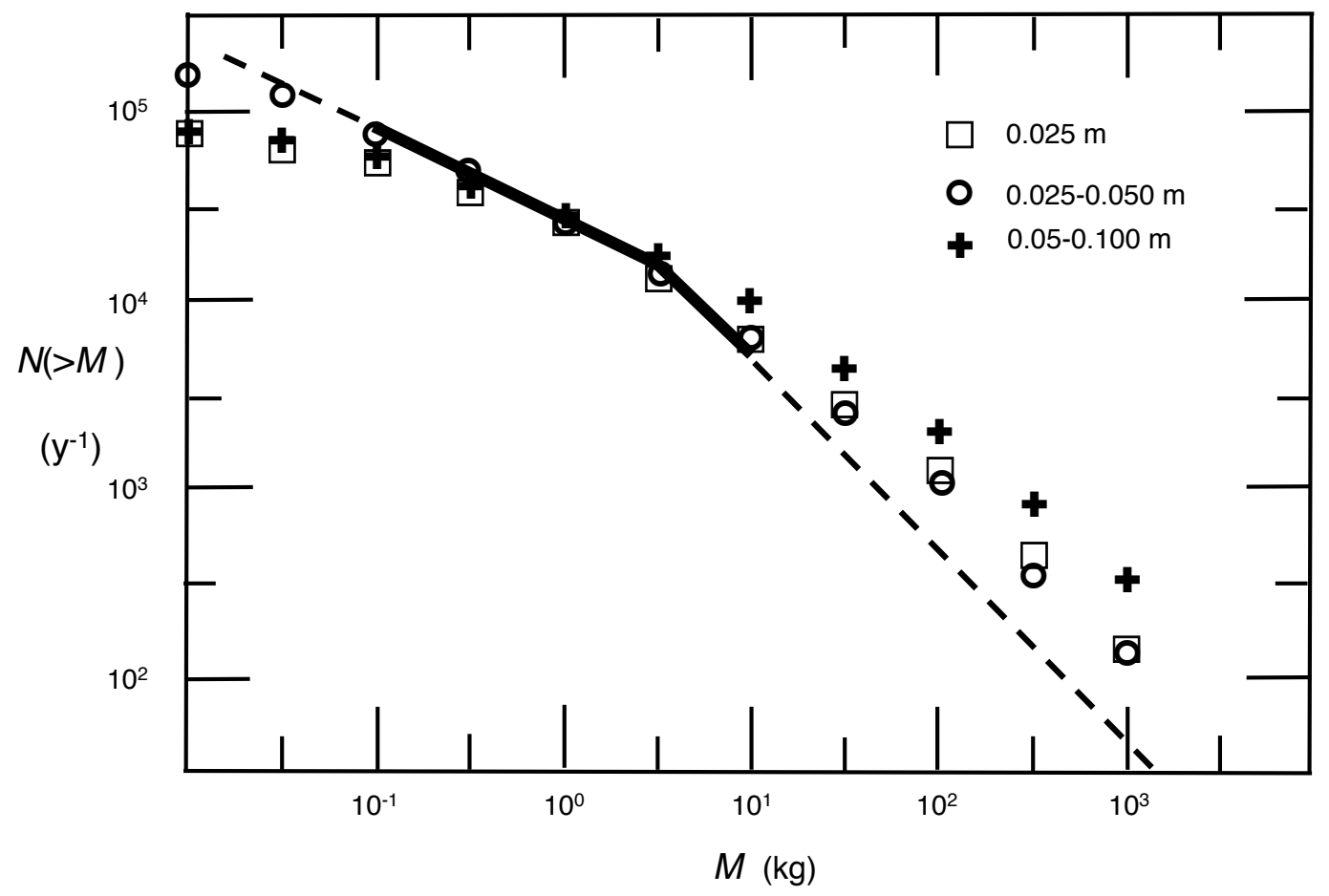

Fig. 1 Cumulative asteroidal meteoroid flux $N(>M)$ at the Earth, where $M$ is meteoroid mass. The heavy broken line for $10^{-1} \mathrm{~kg} \leq M \leq 10^{1} \mathrm{~kg}$ is the data of Halliday et al. (1996, curve AA in their Fig. 1). The dashed lines extend the broken line to lower and higher masses. The squares are for the first space erosion model, which assumes all meteoroids erode by the same amount $\Delta R=0.025 \mathrm{~m}$ in radius before reaching Earth. The circles and crosses are for the second erosion model, which assumes some meteoroids with radii in the range $R+\Delta R_{1}-R+\Delta R_{2}$ erode down to $R$. The circles are for $\Delta R_{1}=0.025 \mathrm{~m}, \Delta R_{2}=0.05$ $\mathrm{m}$, while the crosses are for $\Delta R_{1}=0.05 \mathrm{~m}, \Delta R_{2}=0.10 \mathrm{~m}$. The amplitudes in both models are adjusted so as to approximate the Halliday et al. (1996) data. 




Fig. 2 Schematic diagram illustrating the second space erosion model. Radius $R$ is represented by the vertical line. Some fraction $\kappa$ of the meteoroids with radii between $R+\Delta R_{1}$ and $R+\Delta R_{2}$ (spheres in the upper grey area) erode down to the range $R+d R$ (lower grey area). The same fraction $\kappa$ applies to meteoroids of all radii. 